\title{
Cognitive ultra wideband radio spectrum sensing window length optimization algorithm
}

\author{
Liaoyuan Zeng ${ }^{1 *}$, Sean McGrath² and Guanghui Liu ${ }^{1}$
}

\begin{abstract}
A critical objective of cognitive radio (CR) system is to enhance the spectrum efficiency, and one of the key factors that can determine the spectrum efficiency is the system spectrum sensing performance with respect to sensing window length. For non-coherent detection-based sensing technique, the length of the sensing window required to meet the detection criteria is inversely proportional to the detected signal-to-noise ratio (SNR) of the primary users (PUs). This fact may result in an inadequate use of the white or gray space for the conventional CR system whose transmission and sensing window length are both fixed because a high detected PUs SNR can lead to an excessive long fixed sensing window which occupies the potential CR transmission opportunities while a low received PUs SNR can result in an insufficient sensing window length which degrades the CR detection criteria. In this paper, to improve the spectrum efficiency compared with the fixed sensing/transmission window length-based CR system, we propose an adaptive spectrum sensing window length optimization algorithm. We design the algorithm based on the ultra wideband (UWB) system which is an ideal candidate for the implementation of the CR technology. Based on the analysis of the CR-UWB's spectrum sensing technique in terms of the factors such as spectrum efficiency, spectrum sensing length, PUs SNR, detection criteria etc., we formulate the optimization problem into a convex problem, which enables the proposed algorithm to find the optimal trade-off with low computational complexity between the sensing window length and the desired detection probabilities for the CR-UWB system. Compared with the conventional fixed length spectrum sensing techniques, the proposed algorithm is verified to be able to adapt the length of the CR-UWB's transmission window according to the PUs SNR to optimize the use of the available spectrum while guaranteeing the PUs from being interfered.
\end{abstract}

Keywords: Cognitive radio; Ultra wideband; Spectrum sensing

\section{Introduction}

For cognitive radio (CR) [1], ultra wideband (UWB) can be used as an implementation technology [2]. UWB operates in 3.1 to $10.6 \mathrm{GHz}$ with an extremely low power spectrum density (PSD) of $-41.3 \mathrm{dBm} / \mathrm{MHz}$ [3], which facilitates the underlay spectrum sharing technique to co-exist with the primary users (PUs) that operate within the UWB's wide spectrum band [4]. Figure 1 illustrates the band overlap between the UWB system and the incumbent wireless systems such as wireless LAN [5]. Generally, to protect the PUs from being harmfully interfered, the CR-UWB's

\footnotetext{
${ }^{*}$ Correspondence: lyzeng@uestc.edu.cn

1 School of Electronic Engineering, University of Electronic Science and

Technology of China, 2006 Xiyuan Ave., Chengdu 611731, China

Full list of author information is available at the end of the article
}

transmit power can be decreased to a level that is significantly lower than the UWB's regular PSD, which can result in considerably low spectral efficiency.

By using orthogonal frequency division multiplexing (OFDM) [6], as shown in Figure 2, the UWB system can adaptively adjust the transmit power of the OFDM subcarriers according to the spectrum sensing results within the interested spectrum segments [7]. Figure 2 shows the band allocation scheme in the OFDM-based UWB system. The UWB's operating band is divided into band groups (BGs), each of which contains two or three subbands (SBs), and each SB consists of a series of mutually orthogonal subcarriers, which facilitates the design of dynamic spectrum access (DSA)-based methods. The spectral efficiency of the overlapped spectrum is critical to the overall spectral efficiency of the CR-UWB system and

\section{它 Springer}

C) 2014 Zeng et al: licensee Springer. This is an Open Access article distributed under the terms of the Creative Commons

Attribution License (http://creativecommons.org/licenses/by/2.0), which permits unrestricted use, distribution, and reproduction in any medium, provided the original work is properly cited. 


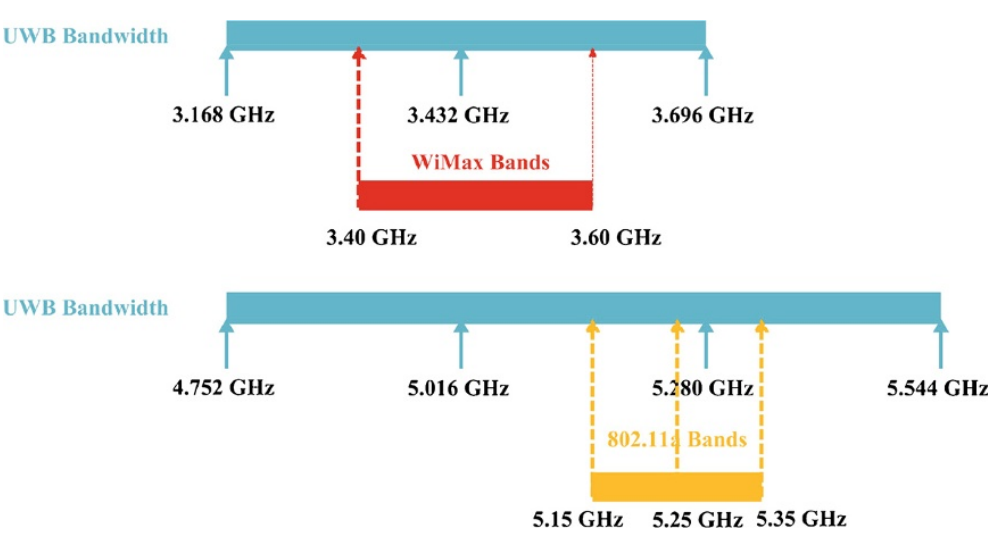

Figure 1 Band overlapping between wireless systems.

is dependent on the performance of the spectrum sensing algorithm [8].

In general cases, the characteristics of the PUs' signal are unknown to the CR-UWB. Thus, the non-coherent detection-based energy detection (ED) technique can be chosen to verify the availability of the overlapped spectrum [9]. In addition, ED's use of the Fourier transform (FT) function can be inherent from the OFDMbased UWB system [10]. According to the features of the ED [11], to determine the PUs' presence successfully, the length of the CR-UWB's spectrum sensing window shall be above a certain threshold which is roughly inversely proportional to the PUs signal's signal-to-noise ratio (SNR) received at the CR-UWB and dependent on the thresholds of the probability of false alarm (PFA) and probability of detection (PD).

For a CR-UWB system in which the duration of spectrum access is usually fixed [12], the length of the CRUWB's spectrum sensing window determines the length of the transmission window. An excessive sensing window fulfilling the PUs' protection requirement can produce a shortened transmission window which will limit CRUWB's use of the overlapped spectrum. Figure 3 shows a pattern of time resource allocation for the use of the available spectrum, i.e., the length fixed transmission opportunity (TXOP), in the CR-UWB system. Obviously, a high ratio between the transmission window length and the sensing window length is preferred [13]. Nevertheless, when PUs operate in a low-SNR regime, i.e., the SNR detected at the CR-UWB's receiver is extremely low, the time required to successfully detect the PUs can be excessively long, which can lead to a considerably short transmission window and may result in an unacceptable spectral efficiency.

In this paper, we propose a spectrum sensing optimization algorithm in low-SNR regime, aiming at improving the spectral efficiency by finding the trade-off optimality of the spectrum sensing window length and the spectrum sensing performance. Followed by an optimized way of effective use of the available spectrum, the proposed

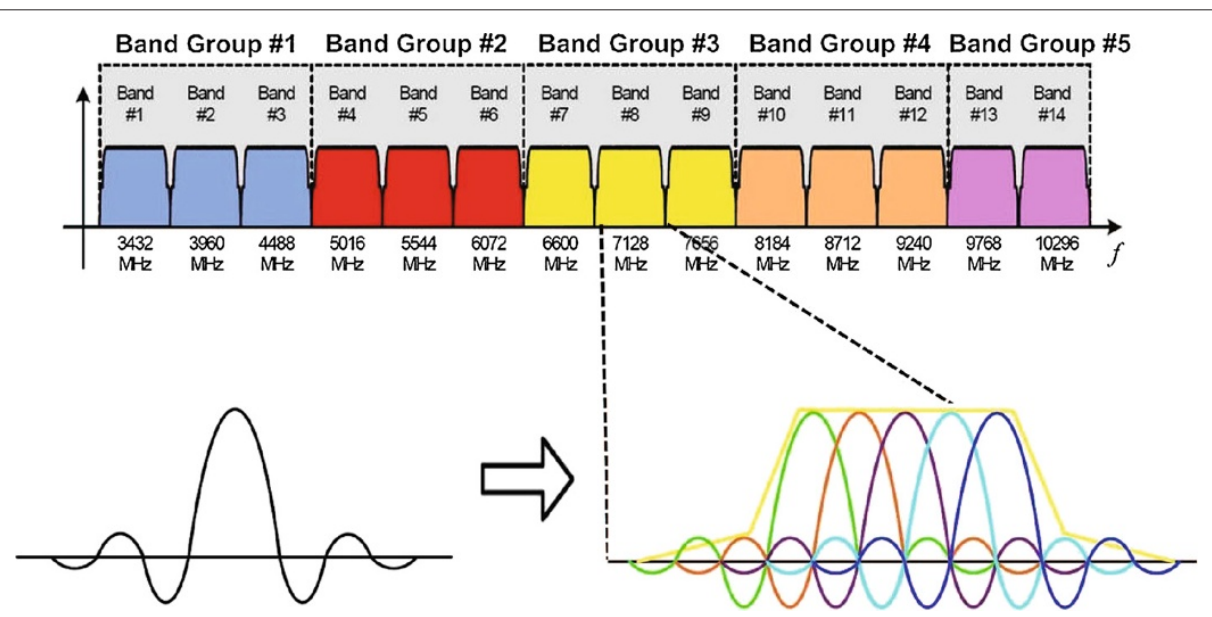

Figure 2 Band allocation of multiband OFDM-based UWB system. 


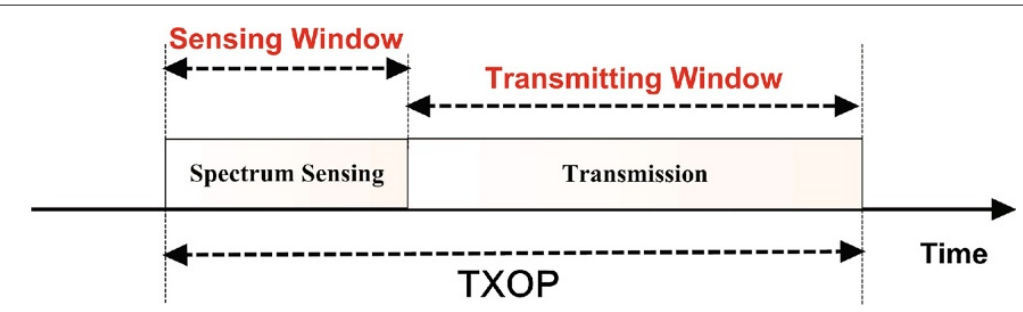

Figure 3 Time allocation in CR-UWB.

algorithm can maximize the proportion between the CR-UWB's transmission window length to the duration of the spectrum access while guaranteeing the PUs' operation.

The remainder of the paper is organized as follows. The 'Literature review' section gives a brief overview of the current spectrum sensing window optimization methods. The next section presents the spectrum sensing model and the formulation of the spectrum sensing window optimization problem. Then, the proposed sensing window optimization algorithm is demonstrated. Furthermore, the performance of the proposed algorithm is verified through numerical simulation which is in the section of numerical results. Finally, we give a conclusion to the paper in the final section.

\section{Literature review}

CR technology can be used in many areas $[5,14,15]$. Specifically, Stotas and Nallanathan and Peh et al. laid the fundamental work for dealing with the spectrum sensing window optimization problem in $[16,17]$, respectively. The Lagrange dual optimization method was used by Stotas and Nallanathan in [16] to optimize the power distribution mechanism and the spectrum sensing scheme by tuning the sensing window length adaptively to optimize the throughput of the CR system. In Stotas and Nallanathan's algorithm, the iterations required for identifying the multiple Lagrange multipliers' values are considerable. In [18], Zou et al. used the Taylor approximation to find the relationship of the PFA and PD. The authors then minimized the CR's overall outage probability through optimizing the sensing window length using linear programming technique [19]. However, the performance of Zou's algorithm in low PU SNR regime will be degraded considerably because the required sensing window length would be excessive.

\section{System model}

In the work, we assume that there exists a heterogenous network consisting of a single user CR-UWB network and a multiuser PU network, as shown in Figure 4. The single CR-UWB network contains one CR communication link, and the CR-UWB's infrastructure is in distributed manner, i.e., ad hoc manner. The CR-UWB network overlaps with the PU network, in which the multiple PUs operate in the same band of the CR-UWB and within the range of being interfered by the CR-UWBs. We assume the use of the overlay spectrum sharing scheme [5], which indicates that the CR-UWB can access to the overlapped spectrum if and only if the CR-UWB's ED result shows that the PUs are temporarily absent. We also assume that an ideal notch filter is used in the CR-UWB system, hence the impact of the sideband interference can be ignored [20].

\section{CR-UWB transceiver}

Figure 5 depicts the architecture of CR-UWB transceiver. It shows that the spectrum sensing function is located in the cognitive engine block which lies posterior to the fast Fourier transform (FFT) engine of the CR-UWB's receiver side. The sensing results can be forwarded into the cognitive algorithm block for the CR-UWB system to dynamically use a radio resource allocation algorithm according to the sensing output.

Furthermore, for spectrum sensing in low-SNR regime, the low noise amplifier (LNA) and the automatic gain control (AGC) at the CR-UWB receiver side are enhanced compared with the conventional UWB transceiver [21]. Since the power of PUs' signals are much higher than that of the UWB user, which will lead to unlinear distortion in LNA, the original LNA is changed to an LNA whose dynamic range is wide enough to handle the power range of primary users' signals. Furthermore, due to the low transmit power of the CR-UWB, the AGC that is set for measuring the peer CR users' signals cannot detect the PUs signals. Hence, the AGC is adjusted according to the number of bits available in the analog-to-digital converter (ADC), so that the CRUWB receiver can detect a wide variety of PUs power levels.

\section{Spectrum sensing method}

Our objective is to find the minimum value of the sensing window length in order to enhance the spectrum efficiency of the overlapped spectrum, in such a way that the time length for the effective data transmission 


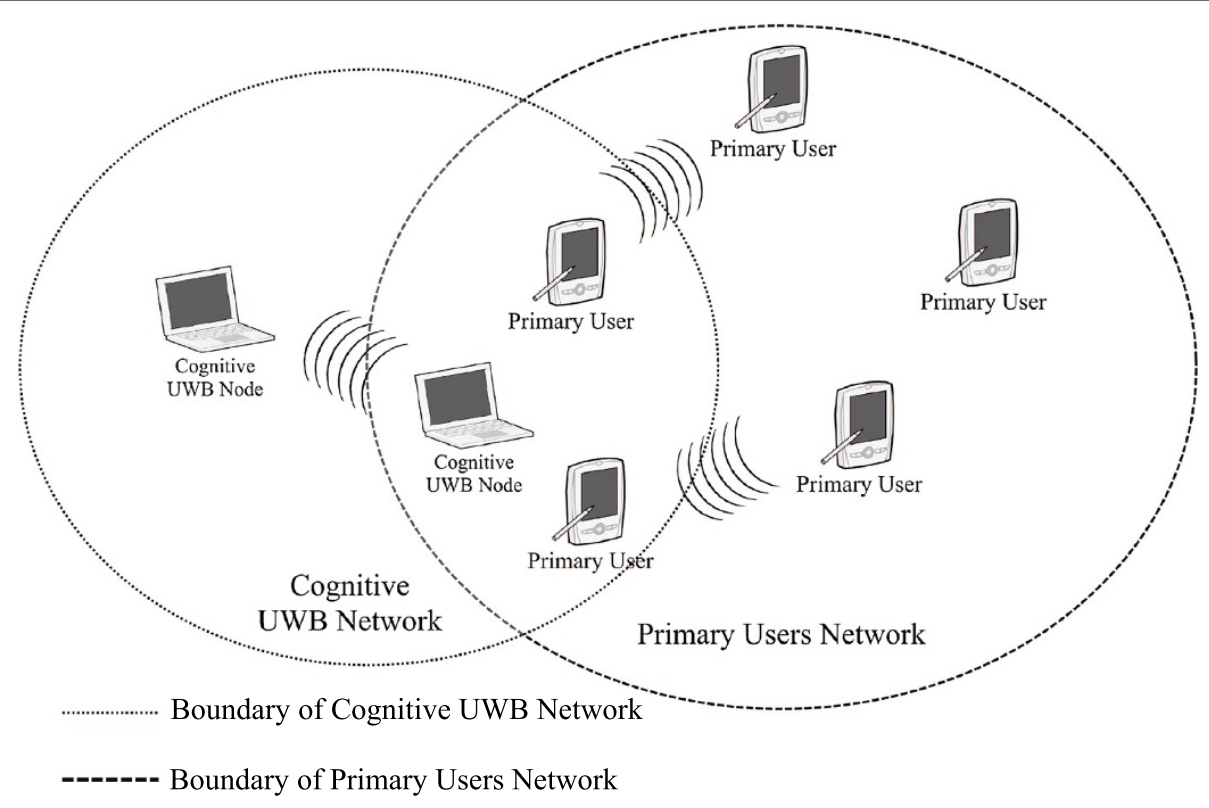

Figure 4 Illustration of a CR-UWB network with multiple PUs.

is maximized. Meanwhile, the value of the sensing window length needs to be large enough for the CR-UWB's ED to successfully determine the availability of the overlapped spectrum. An ED is implemented by averaging the squared magnitude of the K-point FFT of the received signal over the sensing period $T$ which is proportional to the number of received signal samples. Given a fixed point FFT, the increase of the sensing period will improve the estimate of the signal energy [22]. The test statistic for an ED can be given by [23]

$$
T(y)=\frac{1}{N} \sum_{n=1}^{N}|y(n)|^{2}
$$

where $N$ denotes the number of received signal samples, and $y(n)=x(n)+u(n), n \in[1, N]$, represents the discrete received signal at the CR user. Furthermore, $u(n)$ represents the noise and is a Gaussian independent and identically distributed (i.i.d.) random process with mean zero and variance $E\left[|u(n)|^{2}\right]=\sigma_{u}^{2}$. The PU's signal $x(n)$

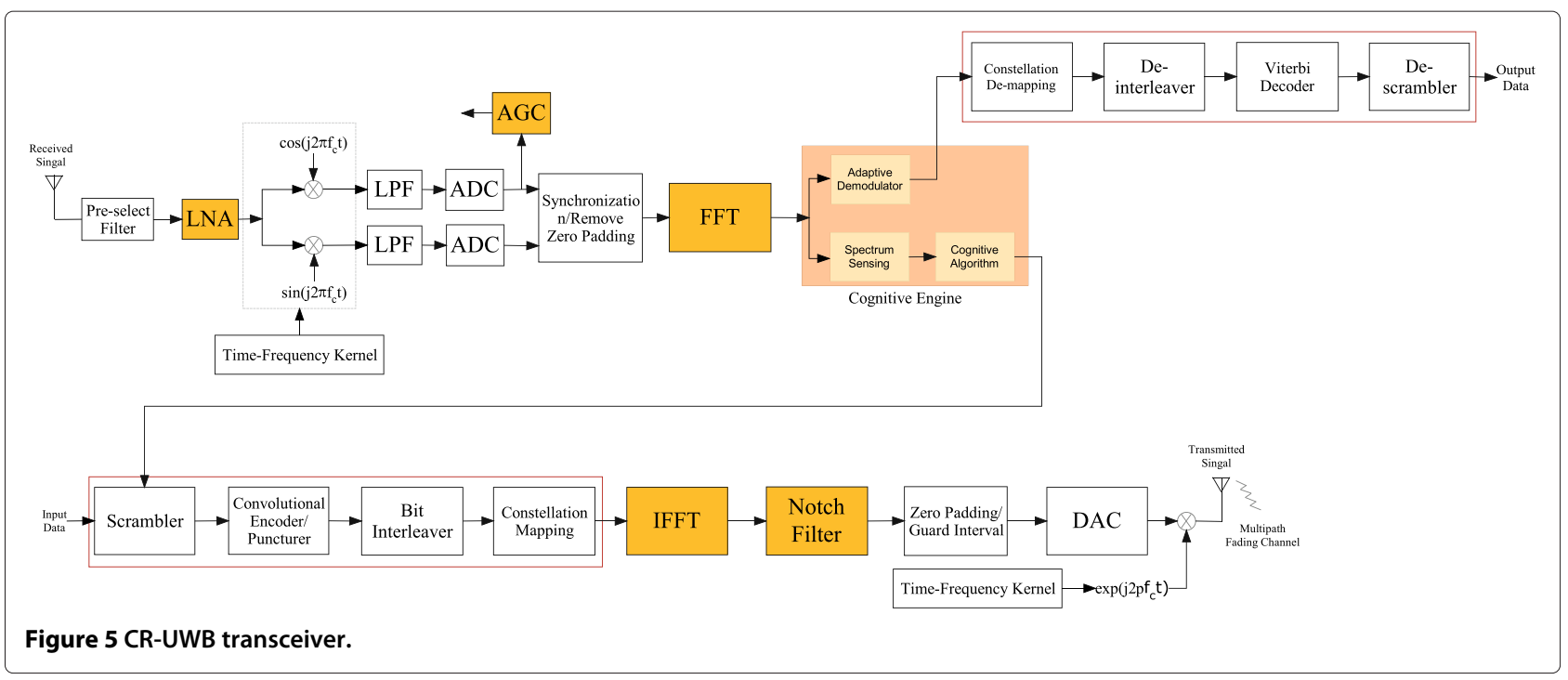


is an i.i.d. random process with mean zero and variance $E\left[|u(n)|^{2}\right]=\sigma_{x}^{2}$, and the parameter $\gamma_{p}=\sigma_{x}^{2} / \sigma_{u}^{2}$ denotes the received SNR of the PU.

The sensing result is determined by comparing $T(y)$ with the threshold, as

$$
\begin{cases}\mathcal{H}_{1}, & T(y)>\epsilon(N) \\ \mathcal{H}_{0}, & T(y)<\epsilon(N)\end{cases}
$$

where $\epsilon(N)$ represents the threshold, $\mathcal{H}_{1}$ denotes the hypothesis that the primary user is present, whereas $\mathcal{H}_{0}$ shows the hypothesis that the primary user is absent.

In an ED, the PD is the probability of detecting under the hypothesis $\mathcal{H}_{1}$ and is determined by the received signal's SNR, the threshold, and the number of signal samples [24]. Thus,

$$
\begin{aligned}
P_{d} & =P\left(T(y)>\epsilon(N) \mid \mathcal{H}_{1}\right) \\
& =P\left(\frac{T(y) / \sigma_{u}^{2}-N-\gamma_{p}}{\sqrt{2\left(2 \gamma_{p}+N\right)}}>\frac{\epsilon(N) / \sigma_{u}^{2}-N-\gamma_{p}}{\sqrt{2\left(2 \gamma_{p}+N\right)}} \mid \mathcal{H}_{1}\right) \\
& \approx Q\left(\frac{\epsilon(N) / \sigma_{u}^{2}-N-\gamma_{p}}{\sqrt{2\left(2 \gamma_{p}+N\right)}}\right) .
\end{aligned}
$$

For PF which denotes the probability of detecting that the licensed user under the hypothesis $\mathcal{H}_{0}$, the expression can be given as [24]

$$
\begin{aligned}
P_{f} & =P\left(T(y)>\epsilon(N) \mid \mathcal{H}_{0}\right) \\
& =P\left(\frac{T(y) / \sigma_{u}^{2}-N}{\sqrt{2 N}}>\frac{\epsilon(N) / \sigma_{u}^{2}-N}{\sqrt{2 N}} \mid \mathcal{H}_{0}\right) \\
& \approx Q\left(\frac{\epsilon(N) / \sigma_{u}^{2}-N}{\sqrt{2 N}}\right) .
\end{aligned}
$$

Hence, for a given target $P_{d}$ and $P_{f}$, the minimum $N$ is determined by manipulating Equations 3 and 4, as

$$
N \approx \frac{2}{\gamma_{p}^{2}}\left[Q^{-1}\left(P_{f}\right)-Q^{-1}\left(P_{d}\right)\right]^{2} .
$$

Note that the complexity of ED is proportional to $\mathcal{O}\left(\frac{1}{\gamma_{p}^{2}}\right)$.

By observing Equation $5, Q^{-1}\left(P_{d}\right)$ and $Q^{-1}\left(P_{f}\right)$ are expressed as

$$
\begin{aligned}
Q^{-1}\left(P_{d}\right) & =\frac{\epsilon(N) / \sigma_{u}^{2}-N-\gamma_{p}}{\sqrt{2\left(2 \gamma_{p}+N\right)}}, \\
Q^{-1}\left(P_{f}\right) & =\frac{\epsilon(N) / \sigma_{u}^{2}-N}{\sqrt{2 N}} .
\end{aligned}
$$

Thus, $P_{d}$ and $P_{f}$ are related to each other by

$$
P_{d}=Q\left(\frac{Q^{-1}\left(P_{f}\right) \sqrt{2 N}-\gamma_{p}}{\sqrt{2\left(2 \gamma_{p}+N\right)}}\right),
$$

$$
P_{f}=Q\left(\frac{Q^{-1}\left(P_{d}\right) \sqrt{2\left(2 \gamma_{p}+N\right)}+\gamma_{p}}{\sqrt{2 N}}\right) .
$$

\section{Problem formulation}

In the work, we model the spectrum sensing window optimization problem as a convex optimization problem. The decision variable is the CR-UWB's sensing window length, and the main constraints consist of the thresholds of CRUWB's PD and PFA. Hence, the problem of optimizing the spectrum sensing window length is expressed as:

$$
\mathbf{P 1} \tau_{s}=\arg \max _{\tau_{s}} \beta S\left(1-P_{f}\right)\left(1-P\left(\mathcal{H}_{1}\right)\right)
$$

subject to

$$
P_{f}=Q\left(\frac{Q^{-1}\left(P_{d}\right) \sqrt{2\left(2 \gamma_{p}+\tau_{s} f_{s}\right)}+\gamma_{p}}{\sqrt{2 \tau_{s} f_{s}}}\right) \geq \overline{P_{f}},
$$

where $S$ represents the spectral efficiency when the CRUWB has full access to the overlapped spectrum where no $\mathrm{PU}$ is surrounded, $\beta$ denotes the ratio between the CRUWB's transmission window length and the fixed duration of the CR-UWB's access to the overlapped spectrum, as

$$
\beta=\frac{\left(T_{\mathrm{op}}-\tau_{s}\right)}{T_{\mathrm{op}}},
$$

where $T_{\mathrm{op}}$ is the pre-defined length of the spectrum access window [12]. The constraint presents the threshold PAF of the CR-UWB's energy detector. The parameter $\tau_{s}$ represents the sensing window length which is determined by the PU's SNR, UWB's sampling frequency, and the target PFA; $P_{f}$ and $\bar{P}_{f}$ represents the actual PFA and the target PFA, respectively, $P_{d}$ represents the real-time PD, and $P\left(\mathcal{H}_{1}\right)$ shows the probability that a $\mathrm{PU}$ activates within $T_{\text {op. }}$.

We assume that the probability that a PU is activated during $T_{\mathrm{op}}$ follows a Poisson process and is expressed as [25]

$$
P\left(\mathcal{H}_{1}\right)=p(x ; \lambda t)=\frac{e^{-\lambda t}(\lambda t)^{x}}{x !},
$$

where $x$ denotes the expected number of occurrences of PU's activations during the period of $t$ which in our system model $t=T_{\mathrm{op}}$.

The objective function in Equation 10 shows that the spectrum efficiency of the overlapped spectrum is determined by multiplying $S$ by the ratio of the CRUWB's transmission window length to the total operating window length when the PU is not activated (i.e., 1 $P\left(\mathcal{H}_{1}\right)$ ), and the CR-UWB's energy detector successfully determines the absence of the PU within the overlapped spectrum (i.e., $1-P_{f}$ ).

For the constraints expressed in $\mathbf{P 1}, \gamma_{p}$ represents the received PU signals SNR at the CR-UWB receiver, and $f_{s}$ 
denotes the UWB's sampling rate. Furthermore, $Q(x)$ is the one-dimensional Gaussian Q-function, as [26]

$$
Q(x)=\int_{x}^{\infty} \frac{1}{\sqrt{2 \pi}} \exp \left(-\frac{y^{2}}{2}\right) d y,
$$

For a certain threshold $\bar{P}_{f}$, the corresponding threshold sensing window length $t \overline{a u} u_{s}$ can be determined by manipulating Equation 5, as

$$
\tau_{s} \geq \frac{2}{\gamma_{p}^{2} f_{s}}\left(Q^{-1}\left(\bar{P}_{f}\right)-Q^{-1}\left(\bar{P}_{d}\right)\right)^{2},
$$

where $\overline{P_{d}}$ denotes the target PD which can be calculated through the use of the receiver operating characteristic (ROC) curves [11], and it shows that the value of the CRUWB's spectrum sensing window length should not be less than the threshold in order to ensure a successful detection of the availability of the overlapped spectrum. The threshold is determined by the value of $\bar{P}_{f}, \bar{P}_{d}$, the received PU signals SNR $\gamma_{p}$, and the CR-UWB system's sampling rate $f_{s}$.

By observing that both $S$ and $P\left(\mathcal{H}_{1}\right)$ are independent of $\tau_{s}$, we can transform the optimization problem shown in P1 into P2 which is simpler to tackle as

$\mathbf{P 2} \tau_{s}=\arg _{\max _{\tau_{s}}} \beta\left[1-Q\left(\frac{Q^{-1}\left(P_{d}\right) \sqrt{2\left(2 \gamma_{p}+N\right)}+\gamma_{p}}{\sqrt{2 N}}\right)\right]$,

subject to

$$
P_{f}=Q\left(\frac{Q^{-1}\left(P_{d}\right) \sqrt{2\left(2 \gamma_{p}+\tau_{s} f_{s}\right)}+\gamma_{p}}{\sqrt{2 \tau_{s} f_{s}}}\right) \geq \overline{P_{f}},
$$

where $N=\tau_{s} f_{s}$ represents the number of spectrum sensing samples of CR-UWB's energy detector,

Figure 6 shows the time for spectrum sensing deduces exponentially when the received SNR $\gamma_{p}$ increases. At low $\gamma_{p}(<15 \mathrm{~dB})$, the sensing time length is longer when the target value of $P_{f}$ is reduced. For example, for the target $P_{f}=0.10, P_{d}=0.90$, and $P_{f}=0.01, P_{d}=$ 0.99 , the time for spectrum sensing $\tau_{s}$ is 250 and $820 \mu \mathrm{s}$ $\left(1 \mu \mathrm{s}=10^{-6} \mathrm{~s}\right)$, respectively. When the value of $\gamma_{p}$ increases to a higher level, such as over $12 \mathrm{~dB}$, the sensing time difference between the two target values of $P_{f}$ becomes minor.

In Figure 7, it shows that the value of $\alpha$ increases exponentially with the increase of the received SNR $\gamma_{p}$. When $\gamma_{p}$ is low $(<-17.6 \mathrm{~dB})$ for $P_{f}=0.01, P_{d}=0.99$, over $50 \%$ of the transmission opportunity is used for spectrum sensing. Thus, the cognitive UWB system can reach a higher spectrum efficiency if the UWB system totally use the TXOP for transmission on the non-overlapped spectrum (i.e., the remaining 64 subcarriers) than performing the spectrum sensing first in order to use the 128 subcarriers for transmission. When the value of $\gamma_{p}$ continues to

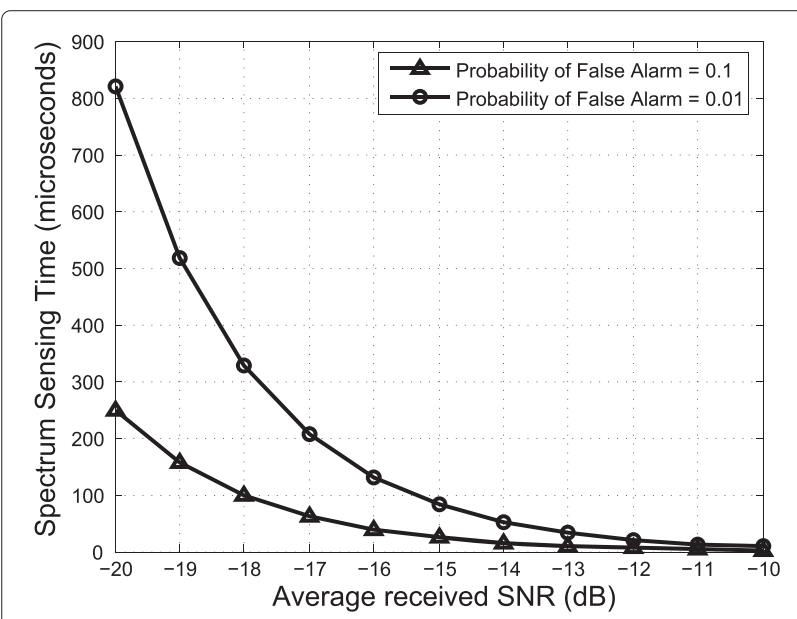

Figure 6 Sensing window length vs. PUs SNR.

increase, the fraction of time differences for UWB's data transmission under the two target values of $P_{f}$ becomes minor.

\section{Sensing window length optimization}

To show the impact of the variation of the spectrum sensing window length on the CR-UWB's spectral efficiency and that there exists an optimal trade-off between the sensing window length and the target PD and PFA, we compute the spectral efficiency of the CR-UWB in the overlapped spectrum as a function of the spectrum sensing window length which is measured in microseconds, as shown in Figure 8.

At the CR-UWB's receiver, when the PUs' signal strength lies in the low-SNR regime, we observe that the spectral efficiency of the CR-UWB grows exponentially with

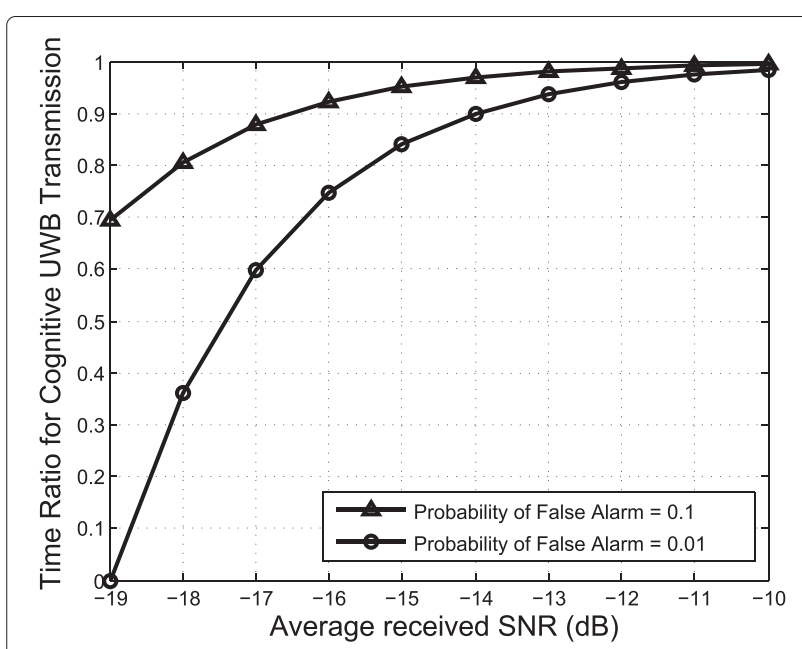

Figure 7 Transmission window length ratio vs. PUs SNR. 


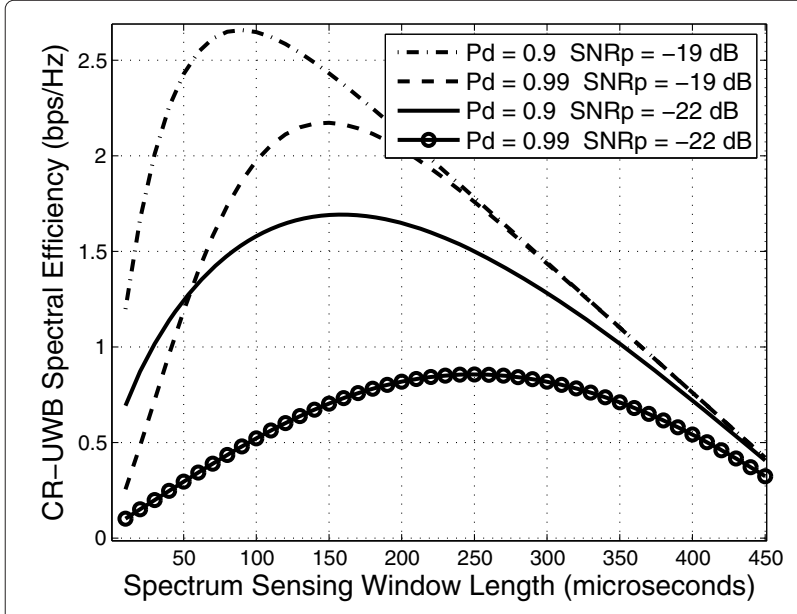

Figure 8 Spectrum efficiency vs. sensing window length.

the increase of $\tau_{s}$ and reaches a peak level when the spectrum sensing window is tuned to a certain value. As the value of $\tau_{s}$ grows beyond the optimal value, the CR-UWB's spectral efficiency decreases monotonically because the transmission window size, $T_{\mathrm{op}}-\tau_{s}$, is shortened. Figure 8 numerically verifies that there exist an optimal $\tau_{s}$ under specific target $\bar{P}_{d}, \bar{P}_{f}$, and $\gamma_{p}$ values.

To compute the optimal spectrum sensing window length for CR-UWB spectral efficiency enhancement under the constrains of $\bar{P}_{f}$ and $\bar{P}_{d}$, we use linear programming to calculate the optimized value of $\tau_{s}$ by computing the root for $f_{r}\left(\tau_{s}\right)=\frac{d F_{r}\left(\tau_{s}\right)}{d \tau_{s}}=0$, where

$f_{r}\left(\tau_{s}\right)=-\frac{1}{T_{\mathrm{txop}}}-\left[Q^{\prime}\left(\tau_{s}\right)-\frac{1}{T}\left(Q\left(f\left(\tau_{s}\right)\right)+Q^{\prime}\left(f\left(\tau_{s}\right)\right)\right)\right]=0$,

where $f\left(\tau_{s}\right)$ represents a function of $\tau_{s}$ and can be expressed as

$$
f\left(\tau_{s}\right)=\frac{Q^{-1}\left(P_{d}\right) \sqrt{2\left(2 \gamma_{p}+\tau_{s} f_{s}\right)}+\gamma_{p}}{\sqrt{2 \tau_{s} f_{s}}}
$$

and the $f\left(\tau_{s}\right)^{\prime}$ s first order differentiation is

$$
\begin{aligned}
f^{\prime}\left(\tau_{s}\right)= & \frac{Q^{-1}\left(P_{d}\right) f_{s}}{2 \sqrt{\left(2 \gamma_{p}+\tau_{s} f_{s}\right) \tau_{s} f_{s}}} \\
& -\frac{\sqrt{2} f_{s}\left(Q^{-1}\left(P_{d}\right) \sqrt{\left(4 \gamma_{p}+2 \tau_{s} f_{s}\right)}+\gamma_{p}\right)}{4\left(\tau_{s} f_{s}\right)^{3 / 2}}
\end{aligned}
$$

Nevertheless, computing the optimal $\tau_{s}$ in closed form is complex. Hence, line search method is used to approximately find the optimal spectrum sensing window length. Figure 9 illustrates the flow diagram of the sensing time optimization algorithm. By initiating the values of $\bar{P}_{f}, f_{s}$ and $T_{\mathrm{op}}$, the target value of $\bar{P}_{d}$ can be identified, which in turn can determine the required $\bar{\tau}_{s}$ that is needed for

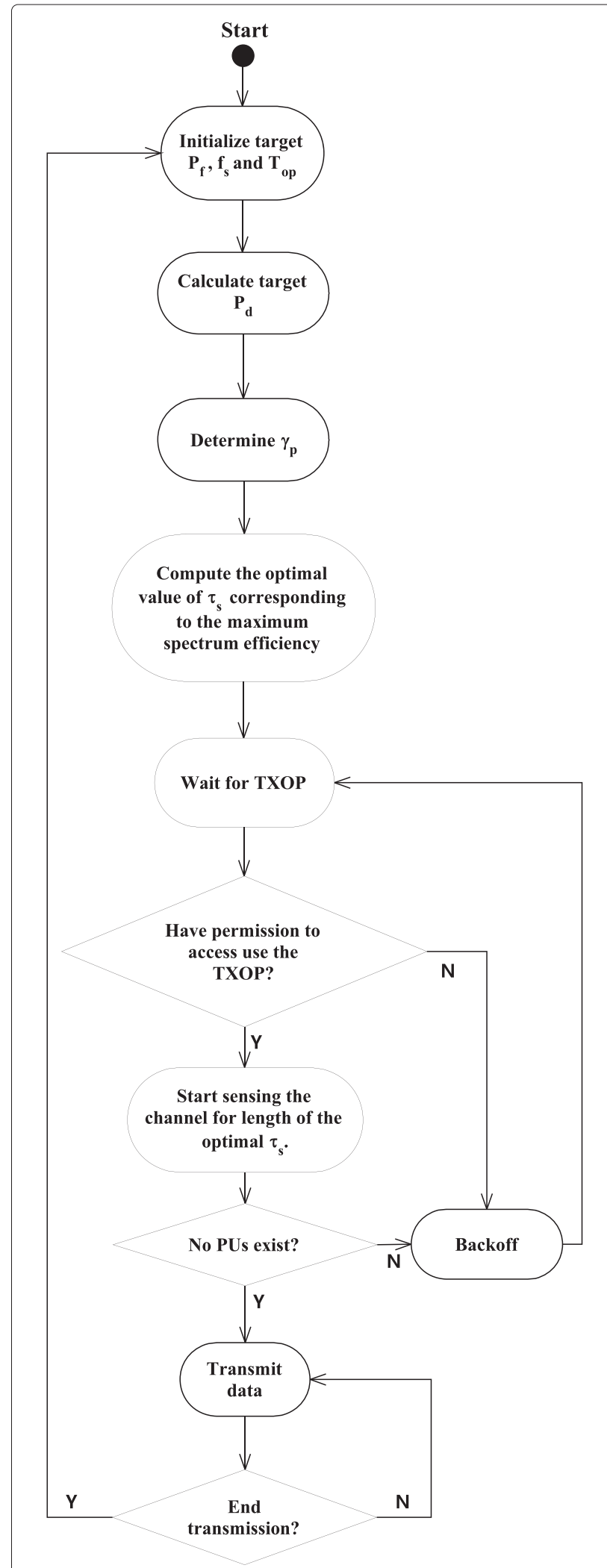

Figure 9 Flow diagram of sensing window length optimization. 


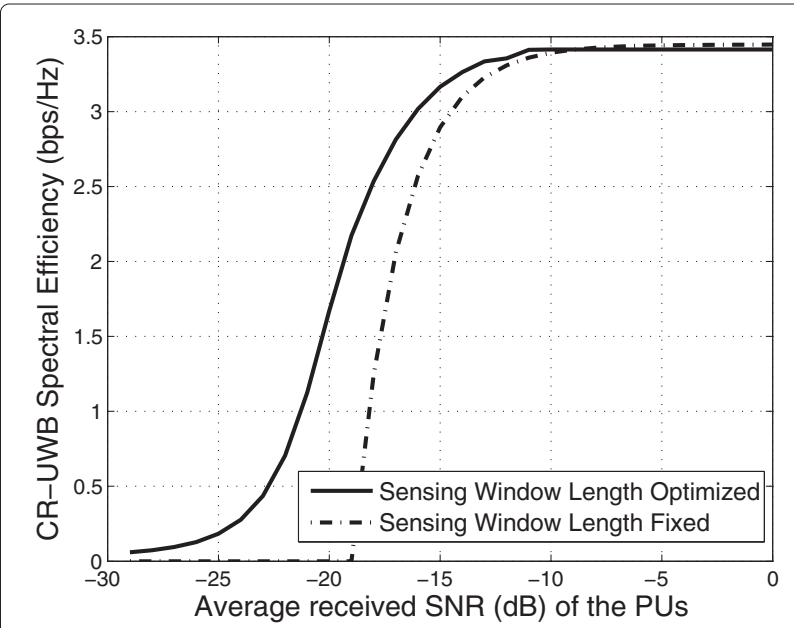

Figure 10 Spectral efficiency vs. $\mathrm{SNR}_{p}$.

successfully detecting the availability of the specific spectrum, under a certain detected value of $\gamma_{p}$. Using line search method, the optimal window length value of $\tau_{s}$ can be found, which can maximize the spectrum efficiency while guaranteeing the operation of the PUs. Then, by successfully accessing the wireless medium through the use of the Carrier sense multiple access with collision avoidance (CSMA/CA)-based MAC scheme [12], the CR-UWB station starts using sensing the spectrum using the optimal $\tau_{s}$. Finally, the sensing results are used by the CR-UWB's transmission function which is shown in Figure 5, to dynamically allocate the radio resource for data transmission, until the transmission period is over.

\section{Numerical simulation}

To validate our algorithm performance in terms of spectral efficiency enhancement, we combine the proposed sensing window length optimization algorithm with the water-filling based transmission scheme proposed in [7]. In our work, the numerical simulation is implemented within a channel model that is previously specified in the UWB system [3]. The detected spectrum hole is used by a water filling-based algorithm [7] where the parameter settings can be referred to. Furthermore, for the PUs, $\lambda$ is set to 1,000 per second to represent the random occupance of the PUs in the overlapped spectrum, and the target PF is set to 0.01 .

We compare the spectral efficiency achieved by the fixed sensing window length-based spectrum sensing method with the proposed sensing window length optimization algorithm, as shown in Figure 10. It is seen that the proposed algorithm can obviously enhance the CR-UWB's spectral efficiency, especially when the PUs operate in low SNR regime. For instance, the corresponding spectral efficiency achieved by the traditional sensing method approaches zero when the value of $\gamma_{p}$ decreases below $-19 \mathrm{~dB}$, while the CR-UWBs spectral efficiency achieved by the proposed sensing algorithm is profoundly higher. The spectral efficiency performance of the proposed algorithm is acceptable even when the PUs SNR decreases below $-25 \mathrm{~dB}$. Furthermore, with the increase of the value of $\gamma_{p}$, the two-lines gap shrinks in an exponential manner. As a comparison, when the PUs operate in normal or high-SNR regime (e.g., $-10 \mathrm{~dB}$ ), the gap becomes minor because a large value of $\gamma_{p}$ can lead to a minor $\tau_{s}$ for a pre-defined threshold of $\bar{P}_{f}$ and $\bar{P}_{d}$.

\section{Conclusion}

To improve the overall spectral efficiency of the OFDM UWB-based CR system when the corresponding PUs' operating power is extremely low, we proposed a novel spectrum sensing window optimization algorithm for the ED-based CR-UWB system to find the optimal tradeoff between the detection probability and the length of the spectrum sensing window. We showed that our algorithm can identify the optimal length of the sensing window length through numerical method constrained by the target PD and PFA and then significantly prolong the transmission window when the duration of the CR-UWB's access to the overlapped spectrum is fixed. By integrating the spectral sensing window optimization algorithm with the existing spectrum management algorithm, the overall spectral efficiency of the CR-UWB was verified to be significantly increased compared with the traditional window length fixed sensing algorithm, especially in PU's low-SNR regime.

Competing interests

The authors declare that they have no competing interests.

\section{Acknowledgements}

Supported by Wireless Access Research Centre at University of Limerick, China Postdoctoral Science Foundation (Y02006023601254) and the Fundamental Research Funds for the Central Universities (ZYGX2013J011).

\section{Author details}

${ }^{1}$ School of Electronic Engineering, University of Electronic Science and Technology of China, 2006 Xiyuan Ave., Chengdu 611731, China. ${ }^{2}$ Department of Electronic and Computer Engineering, University of Limerick, Limerick, Ireland.

Received: 27 September 2013 Accepted: 6 January 2014 Published: 28 January 2014

\section{References}

1. J Mitola III, Cognitive radio for flexible mobile multimedia communications, in Proceedings of the IEEE International Workshop on Mobile Multimedia Communications, San Diego, 15-17 Nov 1999

2. D Porcino, $\mathrm{H}$ Walter, Ultra-wideband radio technology: potential and challenges ahead. IEEE Commun. Mag. 41, 66-74 (2003)

3. L Yang, GB Giannakis, Ultra-wideband communications: an idea whose time has come. IEEE Signal Process. Mag. 21, 26-54 (2004)

4. IF Akyildiz, WY Lee, MC Vuran, Next generation/dynamic spectrum access/cognitive radio wireless networks: a survey. Comput. Netw. 50, 2127-2159 (2006) 
5. E Hossain, V Bhargava, Cognitive Wireless Communication Networks (Springer, New York, 2007)

6. GH Liu, W Zhu, Compensation of phase noise in ofdm systems using an ici reduction scheme. IEEE Trans. Broadcasting. 50(4), 399-407 (2004)

7. LY Zeng, Grath MCS, E Cano, Rate maximization for multiband ofdm ultra wideband systems using adaptive power and bit loading algorithm, in Proceedings of the 9th Advanced International Conference on Telecommunications, Venice, 24-28 May 2009

8. Spectrum Policy Task Force, Report of the spectrum efficiency working group. Technical report, Federal Communications Commission Spectrum Policy Task Force, Washington, D.C., November 2002

9. H Urkowitz, Energy detection of unkown deterministic signals. Proc. IEEE. 55(4), 523-531 (1967)

10. FF Digham, MS Alouini, MK Simon, On the energy detection of unknown signals over fading channels. IEEE Trans. Commun. 55, 21-24 (2007)

11. T Yucek, H Arslan, A survey of spectrum sensing algorithms for cognitive radio applications. IEEE Commun. Surv. Tutorials. 1(11), 116-130 (2009)

12. WiMedia Alliance, Multiband ofdm physical layer specification. Technical report, WiMedia Alliance, 2009

13. LY Zeng, Grath McS, E Cano, Rate maximization for Multiband OFDM Ultra Wideband systems using adaptive power and bit loading algorithm, in Proceedings of the 5th International Conference on Advanced Telecommunications and Systems, Venice, 24-28 May 2009, pp. 369-374

14. B Fette, Cognitive Radio Technology (Elsevier, Burlington, 2009)

15. HL Li, KH Ngan, A co-saliency model of image pairs. IEEE Trans. Image Process. 20(12), 3365-3375 (2011)

16. S Stotas, A Nallanathan, Optimal sensing time and power allocation in multiband cognitive radio networks. IEEE Trans. Commun. 59, 226-235 (2011)

17. ECY Peh, YC Liang, YL Guan, Optimization of cooperative sensing in cognitive radio networks: a sensing-throughput tradeoff view. IEEE Trans. Vehicular Technol. 58, 5294-5299 (2009)

18. Y Zou, YD Yao, B Zheng, Outage probability analysis of cognitive transmissions: impact of spectrum sensing overhead. IEEE Trans. Wireless Commun. 9, 2676-2688 (2010)

19. DP Palomar, JM Cioffi, AL Miguel, Joint tx-rx beamforming design for multicarrier mimo channels: A unified framework for convex optimization. IEEE Trans. Signal Process. 51, 2381-2401 (2003)

20. C Snow, L Lampe, R Schober, Performance analysis and enhancement of multiband ofdm for uwb communications. IEEE Trans. Wireless Commun. 6(6), 2182-2192 (2007)

21. SM Mishra, Brink Ten S, R Mahadevappa, RW Brodersen, Cognitive technology for ultra-wideband/wimax coexistence, in Proceedings of IEEE International Symposium on Dynamic Spectrum Access Networks, Dublin 17-20 Apr 2007, pp. 179-186

22. D Cabric, A Tkachenko, RW Brodersen, Spectrum sensing measurements of pilot, energy, and collaborative detection, in Proceedings of IEEE Military Communication Conference, Washington, D.C., 23-25 Oct 2006, pp. 1-7

23. YC Liang, Y Zeng, ECY Peh, AT Hoang, Sensing-throughput tradeoff for cognitive radio networks. IEEE Trans. Wireless Commun. 7(4), 1326-1337 (2008)

24. A Sahai, N Hoven, R Tandra, Some fundamental limits on cognitive radio, in Proceedings of Allerton Conference on Communication, Control, and Computing, Monticello, 29 Sept.-1 Oct. 2004, pp. 1-11

25. S Huang, X Liu, Z Ding, Opportunistic spectrum access in cognitive radio networks, in Proceedings of the 27th Conference on Computer Communications, Phoenix, 13-18 April 2008

26. $\mathrm{KV}$ Vogel, $\mathrm{H}$ Risken, Determination of quasiprobability distributions in terms of probability distributions for the rotated quadrature phase. Phys. Rev. 40, 2847-2849 (1989)

doi:10.1186/1687-1499-2014-18

Cite this article as: Zeng et al:: Cognitive ultra wideband radio spectrum sensing window length optimization algorithm. EURASIP Journal on Wireless Communications and Networking 2014 2014:18.

\section{Submit your manuscript to a SpringerOpen ${ }^{\circ}$ journal and benefit from:}

- Convenient online submission

- Rigorous peer review

- Immediate publication on acceptance

- Open access: articles freely available online

- High visibility within the field

- Retaining the copyright to your article

Submit your next manuscript at $\boldsymbol{\wedge}$ springeropen.com 